\title{
DSM-5 Reduces the Proportion of EDNOS Cases: Evidence from Community Samples
}

\author{
Paulo P.P. Machado, PhD $^{1 *}$ \\ Sónia Gonçalves, $\mathrm{PhD}^{1}$ \\ Hans W. Hoek, MD, PhD $2,3,4$
}

\begin{abstract}
Objective: Eating Disorder Not Otherwise Specified (EDNOS) constitute the most common eating disorder among those seeking treatment at eating disorder facilities; they are even more common among persons with eating disorders the community. This study compares the impact of applying the revised diagnostic criteria proposed by the DSM-5 workgroup, and the broad categories for the diagnosis of eating disorders (BCD-ED) proposed by Walsh and Sysko on the prevalence of EDNOS.
\end{abstract}

Method: In two nationwide epidemiological studies the prevalence of eating disorders among female high school ( $n$ $=2,028)$ and university students $(n=$ 1,020 ) was examined using DSM-IV criteria. We used a two-stage design, administering a questionnaire in the first stage and an interview in the second stage.
Results: In the combined samples 118 cases of eating disorders (DSM-IV) were detected, of which 86 were diagnosed as EDNOS (72.9\%). Application of the DSM-5 criteria reduced the number of EDNOS cases to $60(50.8 \%)$ or to $52(44 \%)$, when using a BMI $<18.5$ as cutoff for "significantly low weight" criterion in AN; with the use of BCD-ED criteria, only 5 (4.2\%) cases of EDNOS remained.

Discussion: Proposed criteria set for DSM-5 substantially reduce the number of EDNOS cases. However, the BCD-ED scheme further reduces its proportion, almost eliminating it. (C) 2012 by Wiley Periodicals, Inc.

Keywords: EDNOS; DSM-5; epidemiology; classification

(Int J Eat Disord 2013; 46:60-65)

\section{Introduction}

Although anorexia nervosa (AN) and bulimia nervosa (BN) are the most well-known and bestdescribed eating disorders, most people who seek help for an eating disorder do not meet the full diagnostic criteria for these disorders. Most cases fall into the DSM-IV ${ }^{1}$ category of "Eating Disorders - Not Otherwise Specified" (EDNOS), a diagnostic category used for eating disorders that do not meet the criteria for one of the specific eating disorders (i.e., AN or BN).

\footnotetext{
Accepted 8 June 2012

Supported by Contract grant sponsor: Fundação para a Ciência e a Tecnologia/Foundation for Science and Technology, Portugal; contract grant number: PTDC/PSI-PCL/099981/2008

*Correspondence to: Paulo P.P. Machado, Ph.D., Psychotherapy and Psychopathology Research Unit - CIPsi, School of Psychology, University of Minho, Campus de Gualtar, P-4710 BRAGA, Portugal. E-mail:pmachado@psi.uminho.pt

${ }^{1}$ Psychotherapy and Psychopathology Research Unit-CIPsi, School of Psychology, University of Minho, Braga, Portugal

${ }^{2}$ Parnassia Bavo Academy, Parnassia Psychiatric Institute, The Hague, The Netherlands

${ }^{3}$ Department of Epidemiology, Columbia University, New York

${ }^{4}$ Department of Psychiatry, University of Groningen, Groningen, The Netherlands

Published online 20 July 2012 in Wiley Online Library (wileyonlinelibrary.com). DOI: 10.1002/eat.22040

(C) 2012 Wiley Periodicals, Inc.
}

Eating Disorder Not Otherwise Specified is a category for eating disorders that, according to the DSM-IV, ${ }^{1}$ feature behavior variants such as (1) for females, all symptoms of AN but amenorrhea, (2) all symptoms of AN except that current weight is in the normal range, (3) all the criteria for $\mathrm{BN}$, but the frequency of binges or compensatory behaviors do not meet the frequency criterion, or last less than 3 months, (4) inappropriate compensatory behaviors by individuals of normal weight after eating small amounts of food, (5) repeated chewing and spitting out, but not swallowing large amounts of food, and (6) Binge Eating Disorder: recurrent episodes of binge eating in the absence of other inappropriate regular compensatory behaviors, characteristic of bulimia nervosa.

Recent studies indicate that EDNOS cases account for $40 \%^{2-4}$ to $60 \%{ }^{5-9}$ of those seeking treatment at eating disorder facilities. EDNOS may be even more common in nonspecialized settings ${ }^{10}$ and in the community. ${ }^{11}$

Recent discussions regarding the revision of the criteria for the next edition of the DSM due in 2013 has generated several proposals to address the current limitations of the DSM-IV. For example, Walsh and Sysko ${ }^{12}$ have proposed a set of broad categories for the diagnosis of eating disorders (BCD-ED) that, while reducing the possible number of 
individuals in the EDNOS category, would maintain the traditional diagnostic distinctions. BCD-ED proposes three broadly defined disorders: $\mathrm{AN}$ and Behaviorally Similar Disorders (AN-BSD), BN and Behaviorally Similar Disorders (BN-BSD), and BED and Behaviorally Similar Disorders (BED-BSD). It also includes a residual EDNOS category. Each broad category includes a "prototypical" case and subgroups, which include less typical clinical presentations.

Meanwhile, the American Psychiatric Association $^{13}$ has made the preliminary draft revisions to the current diagnostic criteria for eating disorders diagnoses available for public review (see www.dsm5.org). ${ }^{13}$ Proposed changes include: (1) eliminating amenorrhea as a requirement for the diagnosis of AN; (2) reducing the frequency of recurrent binge-eating episodes and inappropriate compensatory behaviors from twice to once per week over the past 3 months; and (3) the formal recognition of Binge Eating Disorder (BED) as a specific ED. The DSM-5, also, proposes changing the EDNOS designation to FEC-NEC (Feeding and Eating Disorders Not Elsewhere Classified.)

The purpose of this study was to evaluate individuals diagnosed with an EDNOS under DSM-IV criteria to determine the proportion that would be reclassified into a broad category using $\mathrm{BCD}-\mathrm{ED}$ criteria or into AN, BN, or BED using the proposed DSM-5 criteria.

\section{Method}

Two nationwide epidemiological studies in Portugal were used to study the prevalence of eating disorders among female high school and university students based on the use of criteria from the DSM-IV, and the impact of the proposed DSM-5 criteria, ${ }^{13}$ and the BCD-ED proposed by Walsh and Sysko ${ }^{12}$ on proportion of EDNOS cases. A two-stage design has been applied. In the first stage a large sample was screened. Individuals who met the criteria for "possible" cases were contacted and interviewed as part of the second stage of the study.

\section{Procedure}

In the first stage of the study, participants were screened with the EDE-Q. ${ }^{14}$ For the second stage of the study, we selected all participants who met at least one the following criteria: (1) BMI $\leq 17.5$, (2) Scores $\geq 4$ on any of the four EDE-Q subscales; (3) a total EDE-Q score $\geq 4$, or, (4) the presence of dysfunctional eating behaviors (i.e., binge eating episodes, inappropriate weight control methods).
In Stage 2, consenting participants were interviewed with the Eating Disorder Examination (EDE). ${ }^{15}$ Clinical psychologists with clinical experience and training in treating eating disorder patients conducted all interviews.

After the interview, responses were recorded on data sheets and all possible symptoms of eating disorder were registered on a checklist. The research clinicians and the principal investigator of the project (PPM) reviewed all diagnosed cases. All diagnostic decisions were reached in a consensus meeting. Consensus about or agreement with the diagnostic decision was reached if positive confirmation of the symptoms was documented and reported in the interview.

All diagnoses were originally made using the DSM-IV criteria. A case was considered EDNOS if it corresponded to one of the six examples presented by the DSM-IV, ${ }^{1}$ clinical severity was determined by clinically significant EDE scores on the relevant diagnostic items and by assessment of clinical impairment by the interviewer.

Subsequently, on the basis of information from the interview records, individuals, originally diagnosed as EDNOS, were reanalyzed with the provisional criteria of the DSM- $5^{13}$ and the criteria proposed in the BCD-ED. ${ }^{12}$ Previously diagnosed cases of AN and BN kept their diagnosis.

For example, using provisional DSM-5 criteria, an originally diagnosed case of EDNOS would be reclassified as $\mathrm{BN}$ if the binge or purge frequency were once a week for 3 months, or as BED if previously diagnosed as EDNOS using the experimental criteria for BED.

For anorexia nervosa, in the DSM-5 the A-criterion is described as: Restriction of energy intake relative to requirements leading to a significantly low body weight in the context of age, sex, developmental trajectory, and physical health. Significantly low weight is defined as a weight that is less than minimally normal, or, for children and adolescents, less than that minimally expected. To apply DSM-5 criteria of AN we used a rather conservative $\mathrm{BMI}<17.5$ as cut off for significantly low weight. However, and because the DSM-5 will also consider a weight severity rating on a scale from 0 to 4 ; and a mildly low weight for AN (score $=1$ ) is defined as BMI $<18.5$, we will also report the number of DSM-5 AN cases, when the $\mathrm{B}$ and $\mathrm{C}$ criteria are met, but the BMI is $>17.5$ but $<18.5$. Presence of other criteria and features of AN were assessed based on interview (EDE) and self-report (EDEQ) clinically significant ratings. For example, restraint subscale for "Restriction of energy intake relative to requirements."

Applying the BCD-ED scheme, individuals originally diagnosed as EDNOS were classified in one of the broad categories, and then further classified into a subgroup of the broad category. If the symptoms were not consistent with a specific subgroup, the Not Otherwise Classified 
FIGURE 1. Participants at Stages 1 and 2.

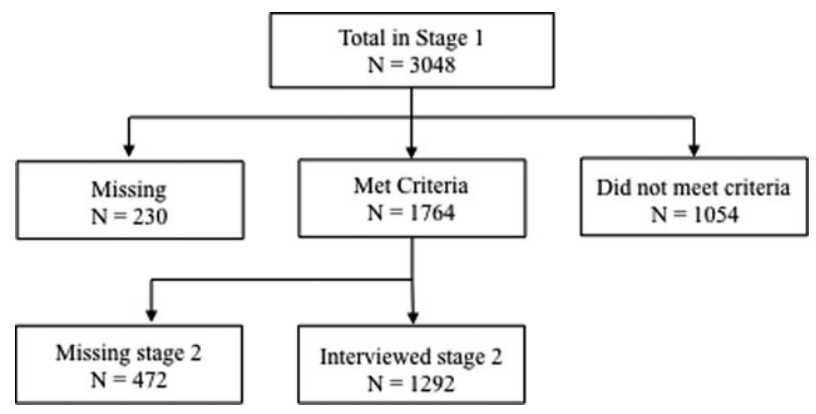

subgroup within the broad category was assigned. Initial reclassifications were made by the second author (SG) and difficult cases were resolved via discussion and additional review of records by the first author (PPM).

\section{Measures}

For stage 1, the Eating Disorder Examination - Questionnaire (EDE-Q, 4th edition) ${ }^{14}$ was used. This is a selfreport questionnaire with 36 items that generates 4 subscale scores (restraint, eating concern, shape concern, and weight concern), as well as a global score, which is the average of the four subscales. Respondents rate each item on a 7-point rating scale (i.e., 0-6) indicating the number of days out of the previous 28 in which particular behaviors, attitudes, or feelings occurred. Scores $\geq 4$ on any of the four EDE-Q subscales were considered clinically relevant. The scale revealed good internal consistency for all the subscales and the Global Score (ranging from 0.71 to 0.97 ).

For Stage 2 the, Eating Disorder Examination (EDE, 12th edition) ${ }^{15}$ was used. This is a researcher-conducted interview developed to measure a broad range of specific psychopathologies characteristic of eating disorders. For this study we used the diagnostic items of the EDE, in order to determine DSM-IV diagnosis. The EDE diagnostic items assess the individual's state in the present and over the previous three months, providing data on the frequency of key behaviors, such us binge eating and self-induced vomiting, and the severity of other features of eating disorders.

\section{Participants}

In the first study, described elsewhere, ${ }^{11} 2028$ high school female students from 11 public schools responded to the EDE-Q. The age of the 2,028 participants ranged from 12 to 23 years; the mean age was $16.19(\mathrm{SD}=1.33$ ) years, and mean BMI was 20.92 (SD = 3.80). For the 1,834 participants screened in the first stage whose responses produced useable data, EDE-Q mean, global, and subscale scores were EDE-Q Restraint, Mean $=0.92$ $(\mathrm{SD}=1.26)$; EDE-Q Eating Concern, Mean = 1.83 (SD $=1.62)$; EDE-Q Shape Concern, Mean =0.65 ( $\mathrm{SD}=0.96)$;
TABLE 1. Eating disorders frequency $(N)$ and prevalence rates (Prev) based on each diagnostic set of criteria for the high school and university samples combined $(N=3,048)$

\begin{tabular}{lccl}
\hline & $\begin{array}{c}\text { DSM-IV } \\
\text { N (Prev) }\end{array}$ & $\begin{array}{c}\text { DSM-5 } \\
\text { N (Prev) }\end{array}$ & $\begin{array}{c}\text { BCD-ED } \\
\text { N (Prev) }\end{array}$ \\
\hline Anorexia nervosa & $18(0.59)$ & $21^{\mathrm{b}}(0.69)$ & $32^{\mathrm{c}}(1.05)$ \\
Bulimia nervosa & $14(0.46)$ & $18(0.59)$ & $62(2.03)$ \\
BED & - & $19(0.62)$ & $19(0.62)$ \\
EDNOS & $86(2.82)$ & $60^{\mathrm{b}}(1.97)$ & $5(0.16)$ \\
Total cases & $118(3.87)$ & $118(3.87)$ & $118(3.87)$ \\
\hline
\end{tabular}

${ }^{a}$ BED cases are included in EDNOS with DSM-IV criteria.

${ }^{b}$ DSM-5 AN BMI< 17.5. If we use a less conservative cutoff point of $\mathrm{BMI}<18.5$ the number will be 29 and the number of EDNOS will be 52 instead of 60 .

' Includes three cases (aged 20, 20, and 21 years) with a BMI of 18.56, $18.57,18.73$ that meet all other DSM5 criteria for AN.

EDE-Q Weight Concern, Mean =1.89 (SD = 0.67); and, EDE-Q Global, Mean = 1.31 (SD = 1.23). In Stage 2, 836 students who met the selection criteria described above were interviewed with the EDE. At Stage 2, using DSM$\mathrm{IV}^{1}$ diagnostic criteria, we detected 62 cases of eating disorders. Of these, 48 cases were classified as EDNOS, corresponding to a prevalence rate of $2.37 \%$. Fourteen cases $(0.69 \%)$ were either anorexia nervosa $(N=8 ; 0.39 \%)$ or bulimia nervosa $(N=6 ; 0.30 \%)$.

In the second study, not previously reported, a sample of 1,020 female university students was screened at Stage 1. Most of the students $(78 \%)$ in this sample were from the North of the country and the remaining from the South. Both campuses are part of the public university system and accept students from all over the country. The age of the 1,020 participants, at Stage 1, ranged from 18 to 58 years, mean age was 21.81 ( $\mathrm{SD}=4.13$ ) years, and mean BMI was 21.99 ( $\mathrm{SD}=3.39$ ). EDE-Q mean, global, and subscale scores for the 1,020 participants who were screened in Stage 1 of the study were EDE-Q Restraint, Mean $=2.26(S D=2.01)$; EDE-Q Eating Concern, Mean $=1.90(\mathrm{SD}=1.80)$; EDE-Q Shape Concern, Mean =2.13 $(\mathrm{SD}=1.85)$; EDE-Q Weight Concern, Mean $=2.21$ (SD =1.74); and, EDE-Q Global, Mean = 2.37 (SD = 1.79). At stage two, 456 of the 670 women who met the selection criteria described above were interviewed. Using DSM$\mathrm{IV}^{12}$ diagnostic criteria, we detected 56 cases of eating disorders. Of these, 38 cases were of cases of EDNOS corresponding to a prevalence rate of $3.72 \%$. Eighteen cases $(1.76 \%)$ were either anorexia nervosa $(N=10 ; .97 \%)$ or bulimia nervosa $(N=8 ; 0.78 \%)$.

\section{Combined Samples}

At Stage 1, 3,048 students from the 11 public high schools and 2 universities that were attending a class at the time the research team visited completed the questionnaires. Some participants (280) did not complete all questionnaires properly, producing missing or unusable data. This corresponds to an attrition rate of $9.2 \%$ at this 
TABLE 2. Demographic characteristics among the 118 individuals with an eating disorder classified using DSM-V, DSM-5, and the broad categories for the diagnosis of eating disorders proposal

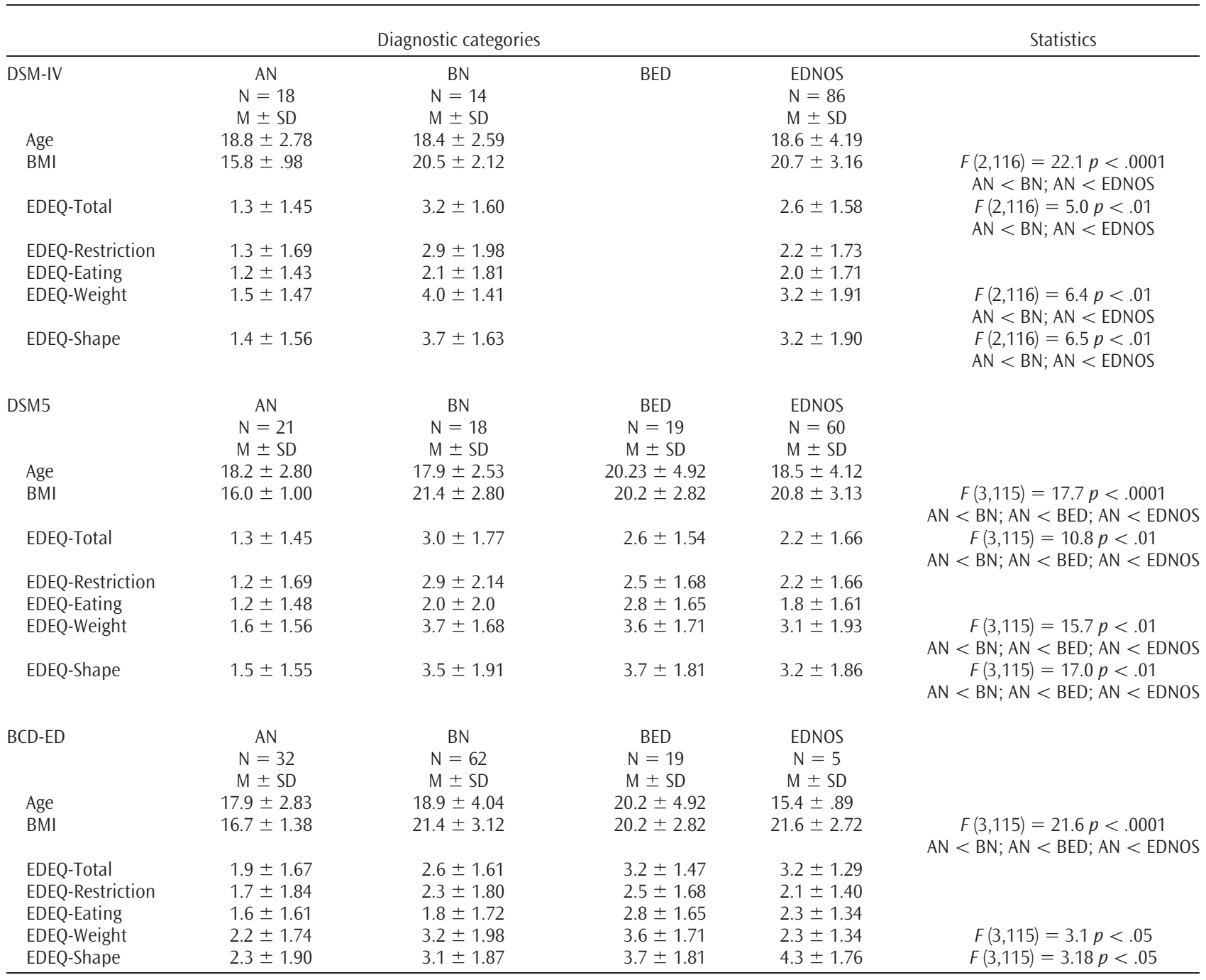

stage. At stage two from the remaining 2,818, 1,764 were selected based on our previously defined criteria. We were able to interview 1,292 participants at Stage 2, and 472 , missed the scheduled interview by either refusing to participate in the interview, failure to obtain consent, or because they missed school that particular day. This corresponds to an attrition rate of $26.7 \%$, at this particular point of the study, representing $15.5 \%$ of the initial sample. Figure 1 presents the number of participants on each stage of the study, as well as the number of missing data at stage one and the number of participants that were interviewed at Stage 2.

\section{Results}

In the combined samples of female high school and university students we detected 118 cases of eating disorders as defined by the DSM-IV, corresponding to a prevalence of $3.87 \%$. Of these, 86 were diagnosed as EDNOS (72.9\% of all cases), 18 as anorexia nervosa (15.3\%), and 14 as bulimia nervosa (11.9\%).

Applying the draft criteria proposed by the DSM$5^{13}$ to the 118 cases diagnosed using DSM-IV criteria, 21 cases $(17.8 \%)$ were classified as anorexia nervosa, 18 (15.3\%) as bulimia nervosa, 19 as binge eating disorder (16.1\%), and the remaining 60 (50.8\%) as EDNOS.

Using the Walsh and Sysco's ${ }^{12}$ broad categories, $32(27.1 \%)$ would be classified as anorexia nervosa and behaviorally similar disorders, 62 (52.5\%) as bulimia nervosa and behaviorally similar disorders, $19(16.1 \%)$ as Binge Eating Disorder and behaviorally similar disorders, and only 5 (4.2\%) as EDNOS. 
TABLE 3. Distribution of the 118 individuals with an eating disorder classified using the broad categories for the diagnosis of eating disorders proposal

\begin{tabular}{lc}
\hline Broad Categories for the Diagnosis of & Total Sample N \\
Eating Disorders (BCD-ED) & 21 \\
\hline Anorexia nervosa and behaviorally similar disorders (AN-BSD) & $(\mathrm{nnn} 1)$ \\
nnn.11, Typical anorexia nervosa, with or without & 0 \\
amenorrhea & \\
nnn.12, Anorexia nervosa, without evidence of & 11 \\
distortions related to body shape and weight & \\
nnn.13, AN-BSD with significant weight loss at or & 0 \\
above a minimally acceptable body weight & 18 \\
nnn.14, AN-BSD-NOS & 25 \\
Bulimia nervosa and behaviorally similar disorders (BN-BSD) (nnn.2) \\
nnn.21, Typical bulimia nervosa & 19 \\
nnn.22, Bulimia nervosa, low frequency & 0 \\
nnn.23, Purging Disorder & 19 \\
nnn.24, BN-BSD-NOS & 0 \\
Binge eating disorder and behaviorally similar disorders (BED-BSD) \\
nnn.31, Typical binge eating disorder \\
nnn.32, Binge eating disorder, low frequency \\
nnn.33, BED-BSD, binge eating episodes not \\
objectively large \\
nnn.34, BN-BSD-NOS \\
Eating Disorder not otherwise specified (EDNOS) (nnn.4) \\
\hline
\end{tabular}

Categories for the diagnosis of eating disorders proposal.

Table 1 shows the prevalence rates, based on the total sample of 3,048 participants, for each eating disorder when DSM-IV, proposed provisional DSM5, and BCD-ED criteria are used. In addition, Table 2 shows the demographic characteristics among the 118 individuals with an eating disorder classified using DSM-V, DSM-5, and the broad categories for the diagnosis of eating disorders proposal. And, Table 3 shows the distribution of clinical cases in each subcategory of eating disorders according to the $\mathrm{BCD}-\mathrm{ED}^{12}$ criteria in each sample and in the combined total sample.

The prevalence of EDNOS cases in the combined samples was $2.79 \%$ using DSM-IV criteria; the prevalence of EDNOS dropped to $1.8 \%$ using proposed DSM- 5 criteria and to $0.2 \%$ using the BCD-ED.

Of the initial 86 EDNOS cases 3 were reclassified as $\mathrm{AN}, 4$ as $\mathrm{BN}, 19$ as $\mathrm{BED}$ using the proposed DSM- 5 criteria; when we used a BMI $<18.5$ as cutoff (instead of $\mathrm{BMI}<17.5$ ) another 8 EDNOS cases were reclassified as DSM-5 AN. When applying the broad categories (BCD-ED), 14 EDNOS cases under DSM-IV criteria were reclassified as AN, 48 as BN and 19 as BED. Figure 2 shows the case migration and the relative proportion of cases under DSM-IV, DSM-5, and BCD-ED criteria.

\section{Discussion}

As expected the prevalence of EDNOS cases in our community sample decreased with the use of both
FIGURE 2. Changes in eating disorder classification by diagnostic classification scheme $(N=118$ cases of eating disorders identified by DSM-IV). AN, anorexia nervosa; BN, bulimia nervosa; BED, binge eating disorder; EDNOS, eating disorder not otherwise specified. Note: BED cases were included in EDNOS under DSM-IV. Subscript numbers represent number of cases that migrated from one to another category. [Color figure can be viewed in the online issue, which is available at wileyonlinelibrary.com.]

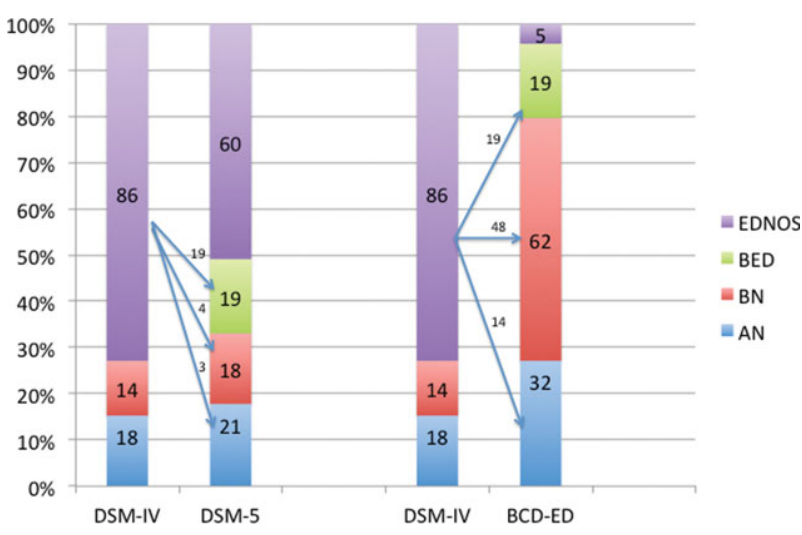

DSM-5 and BCD-ED criteria for the diagnosis of eating disorders. When the DSM5 provisional criteria were applied to the samples the proportion of EDNOS cases identified based on DSM-IV criteria, dropped from $73 \%$ to $51 \%$ (or to $44 \%$, when using a BMI $<18.5$ as cutoff for "significantly low weight" in the A criterion for AN). However, with the use of the proposed BCS-ED criteria, the proportion of EDNOS cases dropped from the original $73 \%$ to $4 \%$.

This suggests that the changes proposed for the DSM-5 significantly reduce the number of EDNOS cases in community samples. However, a significant proportion, corresponding to roughly half of the cases, would still be classified as EDNOS cases or in DSM-5 terminology, FEC-NEC cases.

The proposed BCD-ED criteria almost eliminate the number of EDNOS cases, making it a truly residual category. Although this study suggests that the BCD-ED criteria set has the potential to be clinically useful, it might increase the prevalence rate of cases of eating disorders in the community. This study only reanalyzed cases previously diagnosed as ED under DSM-IV criteria. The broader categories of $\mathrm{BCD}-\mathrm{ED}$ are more heterogeneous, although they seem to capture the essence of each category.

There are several limitations to this study. As noted before, we just reanalyzed previously diagnosed cases under DSM-IV criteria. We did not interview participants again and we relied only notes made during the previous EDE interviews. All previously diagnosed cases of EDNOS were based on examples provided by the DSM-IV there is no 
way to estimate the number of EDNOS in the community based on other examples and/or criteria.

In summary, although the provisional criteria proposed by the DSM-5 Work Group significantly reduce the number of cases of EDNOS previously diagnosed, the proportion of clinical cases in this category remains substantial. A classification scheme based on broader categories of eating disorders such as the BCD-ED would provide major changes in the relative distribution of eating disorders and make EDNOS a truly residual category.

The authors acknowledge Alda Gonçalves and Emilio Juan Compte for their involvement with the data collection.

\section{References}

1. American Psychiatric Association. Diagnostic and Statistical Manual of Mental Disorders-Text Revision (DSM-IV-TR),4th ed. Washington, DC: American Psychiatric Association, 2000.

2. Button EJ, Benson E, Nollett C, Palmer R. Don't forget EDNOS (eating disorder not otherwise specified): Patterns of service use in an eating disorders service. Psychiatric Bull 2005;29: 134-136.

3. Ricca V, Mannucci E, Mezzani B, Di Bernardo M, Zucchi T, Paionni A, et al. Psychopathological and clinical features of outpatients with an eating disorder not otherwise specified. Eating Weight Disorders 2001;6:157-165.
4. Rockert W, Kaplan AS, Olmsted MP. Eating disorder not otherwise specified: The view from a tertiary care treatment center. Int J Eating Disord 2007;40:S99-S103.

5. Fairburn CG, Cooper Z, Bohn K, O'Connor ME, Doll HA, Palmer RL. The severity and status of eating disorder NOS: Implications for DSM-V. Behav Res Therapy 2007;45:1705-1715.

6. Martin CK, Williamson DA, Thaw JM. Criterion validity of the multiaxial assessment of eating disorders symptoms. Int J Eat Disord 2000;28:303-310.

7. Nollett $\mathrm{CL}$, Button EJ. Questionnaire measures of psychopathology in eating disorders: Comparisons between clinical groups. Eur Eat Disord Rev 2005;13:211-215.

8. Turner H, Bryant-Waugh R. Eating disorder not otherwise specified (EDNOS): Profiles of clients presenting at a community eating disorder service. Eur Eat Disord Rev 2004;12:18.

9. Williamson DA, Gleaves DH, Savin SS. Empirical classification of eating disorder not otherwise specified: Support for DSM-IV changes. J Psychopathol Behav Assessment 1992;14:201-216.

10. Zimmerman, Francione-Witt, Chelminski, Young, \&Tortolani, 2008.

11. Machado PPP, Machado BC, Goncalves S, Hoek HW. The prevalence of eating disorders not otherwise specified. Int J Eat Disord 2007;40:212-217.

12. Walsh BT, Sysko R. Broad categories for the diagnosis of eating disorders (BCD-ED): An alternative system for classification. Int J Eat Disord 2009;42:754-764.

13. American Psychiatric Association. Available at:http:// www.dsm5.org/ProposedRevisions/Pages/EatingDisorders.aspx. 2010. Accessed June 23, 2011.

14. Fairburn CG, Beglin SJ. Assessment of eating disorders: Interview or self-report questionnaire? Int J Eat Disord 1994;16:363.

15. Fairburn CG, Cooper Z. The Eating Disorder Examination, 12th ed. In: Fairburn CG, Wilson GT, editors. Binge Eating: Nature, Assessment, and Treatment. New York: Guilford; 1993. p 317. 